\title{
Computer simulation of the rheology of concentrated star polymer suspensions
}

\author{
Johan T. Padding • Evelyne van Ruymbeke • \\ Dimitris Vlassopoulos • Wim J. Briels
}

Received: 6 August 2009 / Accepted: 18 November 2009 / Published online: 9 December 2009

(C) The Author(s) 2009. This article is published with open access at Springerlink.com

\begin{abstract}
We use particle-based computer simulations to study the rheology of suspensions of highfunctionality star polymers with long entangled arms. Such particles have properties which are intermediate between those of soft colloidal particles and entangled polymer chains. In the simulations, each star polymer is coarse-grained to a single particle. In order to faithfully reproduce dynamical properties, it is very important to not only include time-averaged interactions (potentials of mean force) but to also account for transient interactions induced by entanglements between the arms of different star polymers. Using a model which has all these features, it is found that, for sufficiently high shear rates, the start-up shear stress displays an overshoot. With increasing concentration, the core interactions increasingly dominate the initial stress response, leading to a maximum in the stress overshoot at relatively low strain values ( 0.1 to 0.5$)$. Transient forces start to dominate after this initial stage. In a simulated experi-
\end{abstract}

Paper presented at the De Gennes Discussion Conference held February 2-5, 2009 in Chamonix, France.

J. T. Padding $(\varangle) \cdot$ W. J. Briels

Computational Biophysics, University of Twente,

P.O. Box 217, 7500 AE Enschede, The Netherlands

e-mail: J.T.Padding@tnw.utwente.nl

E. van Ruymbeke · D. Vlassopoulos

FORTH, Institute of Electronic Structure and Laser,

Dept. of Materials Science and Technology,

University of Crete, 71110 Heraklion, Crete, Greece

E. van Ruymbeke

Poly, Université catholique de Louvain,

Louvain-La-Neuve, Belgium ment in which the shear rate is suddenly stepped-down from a high to a lower value, the stress shows a clear undershoot, with the minimum stress again at a relatively low strain value (based on the new shear rate). Finally, it is shown that a stress plateau develops in the flow curve. This plateau is absent when the transient forces between the polymer stars are not taken into account.

Keywords Brownian dynamics - Shear flow • Memory effect $\cdot$ Start-up experiment . Stress relaxation $\cdot$ Stochastic simulation

\section{Introduction}

Star polymers are now established as a class of model colloids with tunable interactions, from nearly hard to ultrasoft (Likos 2006; Vlassopoulos 2004; Beris et al. 2008). They consist of a number $(f)$ of linear chains (the arms) covalently joined to a common centre. As such, these colloidal star polymers interpolate between linear polymeric chains and hard colloids; the former case corresponds to $f=1$ or 2 , and the latter to high functionalities $(f \rightarrow \infty)$. It is this wide-ranging weakly repulsive potential that makes star polymers ideal for studying colloidal properties as function of the interactions. Due to their nonuniform monomer density profile (Daoud and Cotton 1982), they are thought of as soft (repulsive) colloidal spheres with a small deformable core and a corona consisting of grafted chains. The interplay of this colloidal and polymeric nature is responsible for a wide range of interesting rheological properties such as glassy, thixotropic, ageing, shear thinning and rejuvenation behaviour. 
Particle-based simulations may help in gaining more insight in the physical mechanisms governing the rheology of star polymer suspensions. Until now, most computer simulations have focussed on the thermodynamic and structural features of star polymer suspensions (von Ferber et al. 2000; Grest et al. 1987; Huissmann et al. 2009; Jusufi et al. 1999, 2001; Likos 2006; Mayer and Likos 2007; Rissanou et al. 2006; Watzlawek et al. 1999), whereas simulations focussing on the dynamics are much more rare and focus mostly on positional correlations (Pakula 1998; Pakula et al. 1998). This paper presents results of the first attempt at simulating the rheology of concentrated solutions of polymer stars of high functionality and long (entangled) arms by a novel computer simulation method called responsive particle dynamics (RaPiD). In order to reach sufficiently large time and length scales, each polymer star is represented by just one particle. It will be shown that, for the dynamics and rheology, it is very important to not only include 'time-averaged' interactions, but to also account for transient interactions induced by 'entanglements' between the star arms. Within our approach, entanglements reflect the difference in free energy of the arms in their non-equilibrium state induced by topological constraints of the overlapping stars. The arms interpenetrate, forming entanglements.

We focus on one particular experimental system, namely, polybutadiene stars with a nominal functionality of 128 arms, each arm having a nominal molar mass $M_{a}$ of around $80 \mathrm{~kg} / \mathrm{mol}$, dissolved in squalene at $T=293 \mathrm{~K}$ (Beris et al. 2008; Helgeson et al. 2007; Roovers et al. 1993). These star polymers have a hydrodynamic radius of $R_{h} \approx 55 \mathrm{~nm}$ (Helgeson et al. 2007), measured by light scattering techniques. The overlap concentration $c^{*}$ is defined as

$c^{*}=\frac{3 f M_{a}}{4 \pi N_{A} R_{h}^{3}}$,

where $N_{A}$ is Avogadro's number, yielding $c^{*} \approx$ $24 \mathrm{mg} / \mathrm{ml}$. Preliminary simulations were performed at $c=c^{*}$, using different settings of the simulation parameters to find the best setting that gives a zeroshear solution viscosity $\left(\eta_{0} \approx 2 \mathrm{~Pa} \mathrm{~s}\right)$ in agreement with experiments at $c=c^{*}$ and a self-diffusion coefficient ( $D \approx 2 \times 10^{-15} \mathrm{~m}^{2} / \mathrm{s}$ ) estimated from the StokesEinstein relation $D=k_{B} T /\left(6 \pi \eta_{0} R_{h}\right)$. Regarding the latter, pulsed-field gradient spin-echo NMR experiments, which can measure the true self-diffusion of a star polymer, find that the long-time self-diffusion decreases strongly not only for $c>c^{*}$, but also in the range $0.5 c^{*} \leq c \leq c^{*}$ (Furukawa et al. 2005). The longtime self-diffusion coefficient at $c^{*}$ is therefore already much smaller than its dilute limit value. This is in agreement with the fact that the observed zero-shear solution viscosity also increases strongly in this range of concentrations; at overlap concentration $c^{*}$, the suspension viscosity $\eta_{0}$ is two orders of magnitude higher than the solvent viscosity $\eta_{s} \approx 0.014 \mathrm{~Pa} \mathrm{~s}$ (Roovers 1994). Before embarking on a programme in which the model is tweaked and optimised to quantitatively predict the rheology of star polymer suspensions, we first want to test whether our model can predict the qualitative features of a star polymer suspension. In the absence of experimental data for the true long-time self-diffusion coefficient for our system, we have used the fact that, at overlap concentration, the system is still more-or-less fluid and assumed that each star polymer moves in a homogenous fluid formed by the other star polymers with a viscosity given by $\eta_{0}$.

In summary, our crude approach may not be entirely correct, and hence, we do not claim full quantitative agreement with the experimental system but rather qualitative agreement. Our expectation is that, as more experimental data become available and as the simulation model will become more refined, the agreement will become more quantitative.

This paper is organised as follows. In the section "Model: responsive particle dynamics simulations", we introduce the simulation model and rationalise the choice of parameters. In the section "Results", we present and discuss our simulation results for the linear and non-linear rheology. We conclude and give an outlook to future directions in the section "Conclusion and outlook".

\section{Model: responsive particle dynamics simulations}

The RaPiD method employed here is based on a classical Brownian dynamics (BD) scheme, but expanded to include positional and temporal memory of the particles (Briels 2009; van den Noort et al. 2007). It allows simulation of slow processes in polymeric systems with great computational efficiency, hence the acronym RaPiD. In this work, the model is specifically constructed to capture the properties of star polymer solutions, yet the same single-particle approach has been used successfully for the simulation of entangled polymers (Kindt and Briels 2007), entangled solutions of polymeric core-shell colloids (van den Noort et al. 2007; van den Noort and Briels 2007, 2008) and transient telechelic polymer networks (Sprakel et al. 2009).

In the model, each star polymer is coarse-grained to only three coordinates, corresponding to the position of the centre of the particle. The effective interaction between two of these particle centres consists of a 
conservative ('star-star') part $V_{s s}$, as in classical BD, and a contribution from transient effects $\Phi_{t}$.

\section{Potential of mean force between star polymers}

The contribution $V_{s s}$ corresponds to the equilibrium interaction potential (the potential of mean force), which has been derived in the past (Likos et al. 1998; Witten and Pincus 1986), and whose validity has been confirmed through comparison with scattering data (Laurati et al. 2005; Likos et al. 1998) and computer simulations (Jusufi et al. 1999). It reads as

$$
\begin{aligned}
\beta V_{s s}(r)= & \frac{5}{18} f^{3 / 2} \\
& \times\left\{\begin{array}{l}
-\ln \left(\frac{r}{\sigma}\right)+\frac{1}{1+\sqrt{f} / 2} \\
\frac{1}{1+\sqrt{f} / 2} \frac{\sigma}{r} \exp \left[-\frac{\sqrt{f}}{2 \sigma}(r-\sigma)\right] \text { else }
\end{array} \text { for } r \leq \sigma\right.
\end{aligned}
$$

Here, $\beta=\left(k_{B} T\right)^{-1}$ and $\sigma$ is the effective corona diameter of the star. Figure 1 (solid line) shows $V_{s s}$ for $f=128$, as used in this paper. The potential shows a soft logarithmic divergence for small distances, followed by a crossover to a Yukawa form as the centre-

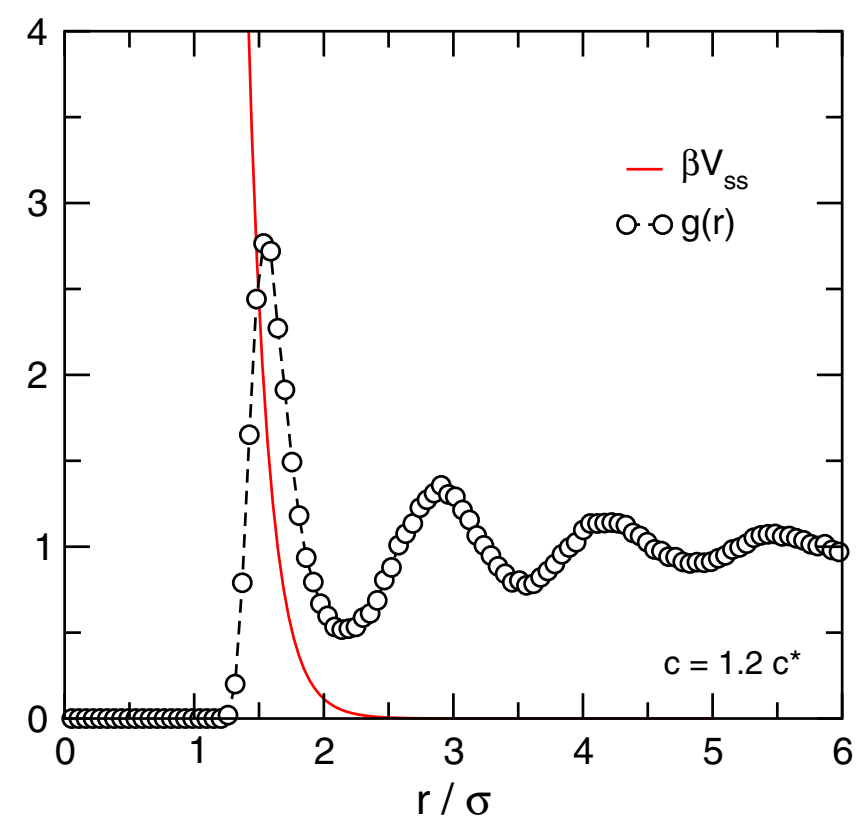

Fig. 1 Equilibrium interaction potential $V_{S S}$ between two star polymers with $f=128$ arms (solid red line) and the radial distribution function $g(r)$ between the centres of the particles at a density $c=1.2 c^{*}$ (circles). Note that pair distances are $1.2 \sigma$ or larger (the first peak is at $1.5 \sigma$ ) to-centre separation grows. In the simulation, the potential is taken to be zero beyond a cut-off distance $r_{c}=3 \sigma$, which is large enough to safely ignore the cutoff error in calculating equilibrium interaction forces, while it is small enough to keep the number of neighbour particles for each particle manageable. Note that the role of many-body forces has also been looked upon by computer simulation and theory (von Ferber et al. 2000), where it was found that they play a negligible role for concentrations up to four to five times the overlap density and, hence, can safely be ignored in this regime.

The corona diameter arises naturally in the blob model for the conformation of isolated stars, introduced by Daoud and Cotton (1982). According to the Daoud-Cotton picture, the bulk of the interior in a star polymer in good solvent conditions (and for sufficiently long arms) consists of a region in which the monomer density profile $c(r)$ follows a power-law as a function of the distance $r$ from the star centre, namely, $c(r) \propto r^{-4 / 3}$ (Jusufi et al. 2001). Outside this scaling region, there exists a diffuse layer of almost freely fluctuating rest chains, in which the Daoud-Cotton scaling is no longer valid. (Note also that, for sufficiently small distances from the centre of the particle, the local monomer concentration will saturate to the melt value. In this work, we consider that the core and this unswollen part of the star can be ignored; see Likos (2006).) Jusufi and co-workers (Jusufi et al. 2001) have shown that a more complete expression for the monomer density profile is given by

$$
\begin{array}{rlr}
c(r)= & A \\
& \times \begin{cases}r^{-4 / 3}(\sigma / 2)^{-5 / 3} & \text { for } r \leq \sigma / 2 \\
\left(\frac{1}{r^{2}}+2 \kappa^{2}\right) \frac{\zeta}{\sigma / 2} \exp \left\{-\kappa^{2}\left[r^{2}-(\sigma / 2)^{2}\right]\right\} & \text { else }\end{cases}
\end{array}
$$

where $\zeta=\left(1+\kappa^{2} \sigma^{2} / 2\right)^{-1}$ and $\kappa$ is a fit parameter of the order of the inverse radius of gyration $R_{g}^{(s)}$ of the star polymer. The prefactor $A$ serves to normalise the monomer density such that its integral yields the total number of monomers in a star polymer. By comparing with density profiles from simulations, it was found that $\kappa R_{g}^{(s)}=0.95$ (Mayer and Likos 2007), and that the link between the radius of gyration of the star and its corona diameter is given by $\sigma \approx \frac{4}{3} R_{g}^{(s)}$. A plot of the monomer density according to Eq. 3 for $f=128$ is given in Fig. 2 (solid line).

Experimentally, the ratio between the hydrodynamic radius and the radius of gyration is given by $R_{h} / R_{g}^{(s)} \approx$ 1.3 over a large range of stars with $f=64$ or 128 


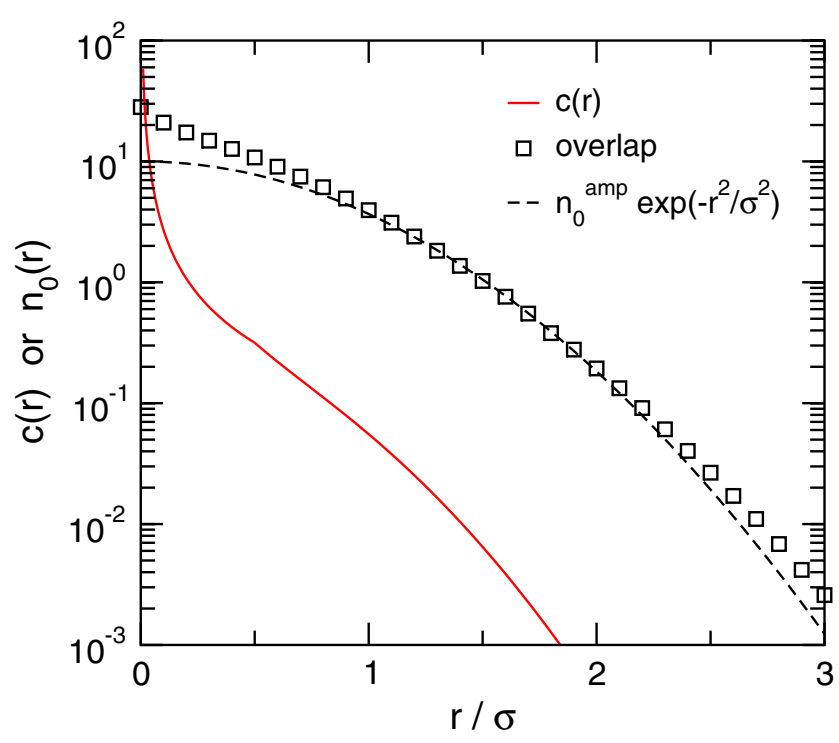

Fig. 2 Monomer density profile $c(r)$ as a function of distance $r$ from the centre of the particle (solid red line), according to Eq. 3, for a star polymer with $f=128$ arms. Numerically calculated overlap integral (squares), representing the functional dependence of the equilibrium number of entanglements between two star polymers at separation distance $r$. Equation 5 (dashed line) gives a reasonable fit in the relevant range of pair distances

(Roovers et al. 1993). Using the experimentally determined hydrodynamic radius of $R_{h} \approx 55 \mathrm{~nm}$, in this work, we choose $R_{g}^{(s)}=42.4 \mathrm{~nm}$ and $\sigma=56.5 \mathrm{~nm}$. Using these numbers, the overlap concentration $c^{*}$ corresponds to 0.27 particles per $\sigma^{3}$.

Transient forces and dynamical properties

The conservative interaction potential $V_{s s}$ given by Eq. 2 determines the equilibrium structural properties of the system. However, it ignores the dynamical effect of entanglements between the arms of the star polymers, which occur predominantly at the scale of the outer blobs (further entanglements are increasingly more difficult) (Daoud and Cotton 1982; Grest et al. 1987; Likos 2006). Imagine, for example, two neighbouring star polymers which are well entangled with each other. If the distance $r_{i j}$ between these star polymers is suddenly increased (e.g. induced by flow), the arms require some time to disentangle. This effect will become increasingly important for star polymers with increasingly long arms. During this time, there will be a transient attractive force between the star polymers. This force originates from the entanglement junctions, and it decays to zero as the arms get disentangled. Therefore, it is envisaged that the transient part $\Phi_{t}$ of the interaction originates from deviations in the actual number of entanglements $n_{i j}$ between two star polymers with respect to the equilibrium number of entanglements $n_{0}\left(r_{i j}\right)$ prevailing at the distance $r_{i j}$. Because, to a first approximation, the number of entanglements is expected to scale with the number of binary contacts between monomers of different star polymers, the equilibrium number of entanglements is assumed to be proportional to the overlap integral between the monomer densities of two star polymers. Placing one star at the origin and the other a distance $r_{i j}$ displaced in the $z$ direction, the overlap can be calculated as:

$n_{0}\left(r_{i j}\right) \propto \int \mathrm{d} \mathbf{r} c(\mathbf{r}) c\left(\mathbf{r}-r_{i j} \hat{\mathbf{e}}_{z}\right)$

The result of a numerical calculation of the overlap integral for $f=128$ is shown in Fig. 2 (squares). The radial distribution function in Fig. 1 (circles) shows that typical pair distances are of the order of $1.5 \sigma$ (the first peak) or larger. This suggests that the nearly Gaussian part of $c(r)$ for $r>\sigma / 2$ will dominate the overlap integral for all relevant distances. Indeed, in the range $r_{i j}>\sigma$, a reasonable fit can be made with a Gaussian dependence (dashed line in Fig. 2):

$n_{0}\left(r_{i j}\right)=n_{0}^{\mathrm{amp}} \exp \left(-\frac{r_{i j}^{2}}{\sigma^{2}}\right)$.

This expression is used in the simulations, with a cutoff at $r_{c}=3 \sigma$. The prefactor $n_{0}^{\mathrm{amp}}$ may be used to set the absolute number of entanglements. However, because only the product of $n_{0}^{2}$ with another parameter ( $\alpha$, as explained in the next paragraph) is relevant, one of the two parameters may be set to an arbitrary value while the other is used to tune the system. Here, $n_{0}^{\text {amp }}$ is set equal to 10 so that $n_{0}\left(r_{i j}\right) \approx 1$ at the typical distance $r_{i j}=$ $1.5 \sigma$ of the closest neighbouring particles. In this way, $n_{0}\left(r_{i j}\right)$ can be interpreted loosely as the fraction of the maximum number of entanglements between a pair of star polymers.

The equilibrium structure determined by $V_{s s}$ is not perturbed if the transient part of the interactions is chosen according to van den Noort et al. (2007):

$\Phi_{t}=\frac{1}{2} \alpha\left(n_{i j}-n_{0}\left(r_{i j}\right)\right)^{2}$.

As alluded to before, we observe that only the product $\alpha n_{0}^{2}(r)$ is of relevance to the interaction $\Phi_{t}$. The parameter $\alpha$ determines the allowed fluctuations of $n_{i j}$ around $n_{0}\left(r_{i j}\right)$ : according to the equipartition theorem, each quadratic contribution to the energy will have an average value of $\frac{1}{2} k_{B} T$; hence, $\left\langle\left(n_{i j}-n_{0}\left(r_{i j}\right)\right)^{2}\right\rangle=$ $k_{B} T / \alpha$. In the simulation, $\alpha$ is set to $4 k_{B} T$. Similar to the case of the conservative interactions, the transient 
interactions are taken to be zero beyond the cut-off distance $r_{c}=3 \sigma$.

A surplus or deficiency in the fraction of entanglements $n_{i j} \neq n_{0}\left(r_{i j}\right)$ relaxes in the simulation as

$\mathrm{d} n_{i j}=-\frac{\left(n_{i j}-n_{0}\left(r_{i j}\right)\right)}{\tau} \mathrm{d} t+\theta \sqrt{\frac{2 k_{B} T \mathrm{~d} t}{\alpha \tau}}$

where the first term represents the tendency of the entanglement fraction to grow or diminish towards the equilibrium value with a characteristic time $\tau$, and the second term gives the noise on the transient forces ( $\theta$ is a number from a univariant normal distribution) (van den Noort et al. 2007). The characteristic time must be relatively large because the long chains in the corona take a long time to entangle or disentangle. In start-up shear experiments at $c=1.5 c^{*}$, overshoot is observed for shear rates $\geq 0.005 \mathrm{~s}^{-1}$ (Beris et al. 2008). Preliminary simulations at $c=1.5 c^{*}$ showed that overshoots at such low shear rates arise only when the relaxation time is set sufficiently large: for $\tau \gg 1 \mathrm{~s}$, all observations and conclusions remain essentially unchanged. In the simulations, $\tau$ is set to $100 \mathrm{~s}$.

The friction $\xi_{i}$ on a particle $i$ for motion relative to the average flow field consists of two contributions:

$\xi_{i}=\xi_{0}+\xi_{e} \sum_{j} \sqrt{n_{i j} n_{0}\left(r_{i j}\right)}$

where $\xi_{0}$ is the dilute limit friction with the solvent and $\xi_{e}$ an additional friction associated with the entanglements with other star polymers. This form ensures that there is no entanglement friction between particles beyond the cut-off $r_{c}$. The value of $\xi_{0}$ is set equal to $6 \pi \eta_{s} R_{h}$, where $\eta_{s} \approx 0.014 \mathrm{~Pa} \mathrm{~s}$ is the solvent viscosity and $R_{h} \approx 55 \mathrm{~nm}$ is the experimentally determined hydrodynamic radius. The value of $\xi_{e}$ is set to $10^{-8} \mathrm{~kg} / \mathrm{s}$. Together with the chosen value of $\alpha$, this predicts a zero-shear viscosity and star self-diff usion coefficient at overlap concentration in agreement with experiment.

\section{Propagator}

Particle positions are updated according to the $\mathrm{BD}$ propagator (van den Noort et al. 2007)

$$
\begin{aligned}
\mathrm{d} \mathbf{r}_{i}= & \left\langle\mathbf{V}\left(\mathbf{r}_{i}\right)\right\rangle \mathrm{d} t+\nabla_{i}\left(k_{B} T / \xi_{i}\right) \mathrm{d} t+\theta^{\prime} \sqrt{2 k_{B} T \mathrm{~d} t / \xi_{i}} \\
& +\frac{1}{\xi_{i}} \sum_{j}\left\{-\nabla_{i} V_{s s}\left(r_{i j}\right)+\alpha\left(n_{i j}-n_{0}\left(r_{i j}\right)\right) \frac{\mathrm{d} n_{0}}{\mathrm{~d} r_{i j}} \frac{\mathbf{r}_{i j}}{r_{i j}}\right\} \mathrm{d} t .
\end{aligned}
$$

Here, $\left\langle\mathbf{V}\left(\mathbf{r}_{i}\right)\right\rangle$ is the average flowfield at position $\mathbf{r}_{i}$ and $\theta^{\prime}$ is a random vector with each component drawn from a univariant normal distribution. The integration time step is chosen equal to $\mathrm{d} t=10^{-6} \mathrm{~s}$. This time step was determined by performing a series of test simulations, finding the largest safe time step for which the equilibrium fluctuations in the entanglement numbers $n_{i j}$ obey equipartition.

Flow in the $x$-direction with a velocity gradient $\dot{\gamma}$ in the $y$-direction is generated by Lees-Edwards boundary conditions (Allen and Tildesley 1987). The $y$-dependence of the (implicit) solvent flowfield in the $x$-direction is dynamically updated by coupling it to the star polymer motion with an averaging time of $10^{-3} \mathrm{~s}$, as described in van den Noort and Briels (2008), Padding et al. (2008). This algorithm allows any $y$-dependent flowfield to form. The simulation box is cubic and always contains $N=729$ star polymers. The exact dimensions depend on the concentration, which assumes values $c^{*}, 1.2 c^{*}$ and $1.5 c^{*}$. For the overlap concentration $\left(c=c^{*}\right)$, each side of the box measures $14 \sigma$.

During the simulations, the instantaneous (microscopic) shear stress $\sigma_{x y}(t)$ is calculated from

$\sigma_{x y}(t)=-\frac{1}{V} \sum_{i<j} x_{i j}(t) F_{y, i j}(t)$,

in which $V$ is the volume of the simulation box, $x_{i j}=$ $x_{i}-x_{j}$ and $F_{y, i j}$ the total force (conservative and transient) in the $y$-direction on particle $i$ due to the presence of particle $j$.

\section{Results}

\section{Linear rheology}

By analysing the fluctuations of the microscopic shear stress in equilibrium simulations, the zeroshear relaxation modulus is obtained from $G(t)=$ $\beta V\left\langle\sigma_{x y}(t) \sigma_{x y}(0)\right\rangle$, where $\sigma_{x y}$ is given by Eq. 10 and the brackets indicate an average over many time origins. Other properties, characteristic of linear rheology, can be derived from $G(t)$. For example, the storage and loss moduli $G^{\prime}(\omega)$ and $G^{\prime \prime}(\omega)$ can be obtained through a Fourier transform, and the zero-shear viscosity is given by the infinite time integral, $\eta_{0}=\int_{0}^{\infty} G(t) \mathrm{d} t$.

Figure 3 (solid lines) shows the shear relaxation modulus for star polymer suspensions at $c=c^{*}, c=$ $1.2 c^{*}$ and $c=1.5 c^{*}$. To make the influence of the transient forces more explicit, the shear relaxation modulus is also calculated from simulations where, instead of dynamically updating the entanglement numbers $n_{i j}$ (Eq. 7), the equality $n_{i j}=n_{0}\left(r_{i j}\right)$ is forcefully imposed (dashed lines). Equations 6, 8 and 9 show that this effectively disables the transient forces, leading to a 


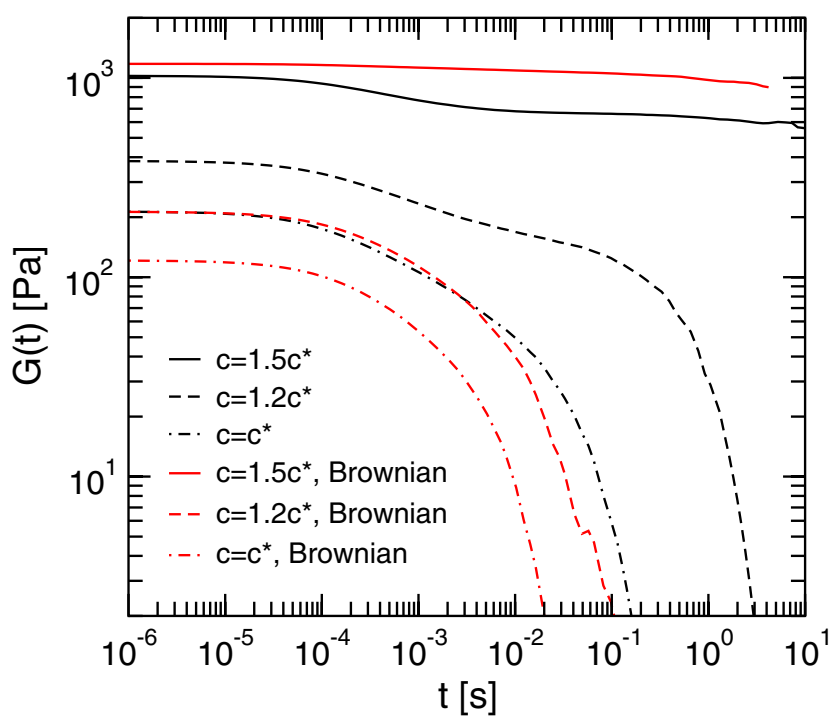

Fig. 3 Shear relaxation modulus for suspensions of star polymers with $f=128$ arms at $c=1.5 c^{*}$ (solid lines), $c=1.2 c^{*}$ (dashed lines) and $c=c^{*}$ (dot-dashed lines) obtained from equilibrium stress fluctuations. Results from simulations with transient forces (black) are compared with results from simulations without transient forces (by setting $n_{i j}=n_{0}\left(r_{i j}\right)$; red). Note the extremely slow (glassy) relaxation of the shear relaxation moduli for $c=1.5 c^{*}$, which are therefore not ergodic

simple BD scheme with the correct order of magnitude for the friction on each particle. Clearly, the transient forces introduce a slow stress relaxation mode, the influence of which grows very rapidly over a relatively small range of concentrations. At $c=c^{*}$, only small deviations from single-exponential relaxation are visible, whereas at $c=1.2 c^{*}$ stress relaxes over two clearly disparate time scales.

For the highest concentration studied here, $c=$ $1.5 c^{*}$, the shear relaxation modulus cannot be reliably obtained, either with or without transient forces. Large stresses are present which do not relax sufficiently over the course of the simulation. This implies a strong dependence on the initially chosen distribution of star polymer positions, so one must not attribute too much meaning to the amplitudes shown in Fig. 3 for $c=$ $1.5 c^{*}$. Previous dynamic light scattering measurements on very similar polybutadiene stars identified the glass transition to occur around $c=1.4 c^{*}$ (Helgeson et al. 2007; Stiakakis et al. 2002a). Inspecting mean-square displacements of the particles (not shown) confirms that our $c=1.5 c^{*}$ system is in a glassy state. Such vitrified star polymer solutions can be liquified by adding amounts of homopolymer chains (Likos 2006; Stiakakis et al. 2002b). Alternatively, the sample may by fluidised by subjecting it to deformation flow (Vlassopoulos 2004; Beris et al. 2008; Helgeson et al. 2007). We will study the response to shear flow in the following sections.

\section{Start-up shear stress}

Figure 4 shows the start-up shear stress measured in a previously glassy sample at $c=1.5 c^{*}$, which is suddenly

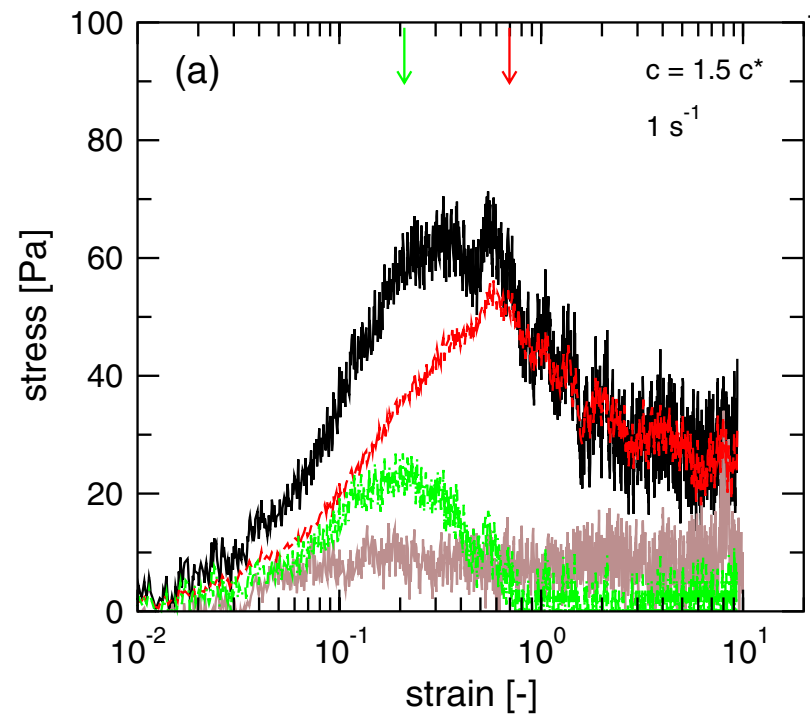

Fig. 4 Start-up shear stress for a star polymer suspension at $c=$ $1.5 c^{*}$ and an applied shear rate of $\mathbf{a} \dot{\gamma}=1 \mathrm{~s}^{-1}$ or $\mathbf{b} \dot{\gamma}=10 \mathrm{~s}^{-1}$. The total stress (solid black line) is a sum of stress originating from conservative forces (dot-dashed green line) and stress originating from transient forces (dashed red line). Note that the stress due

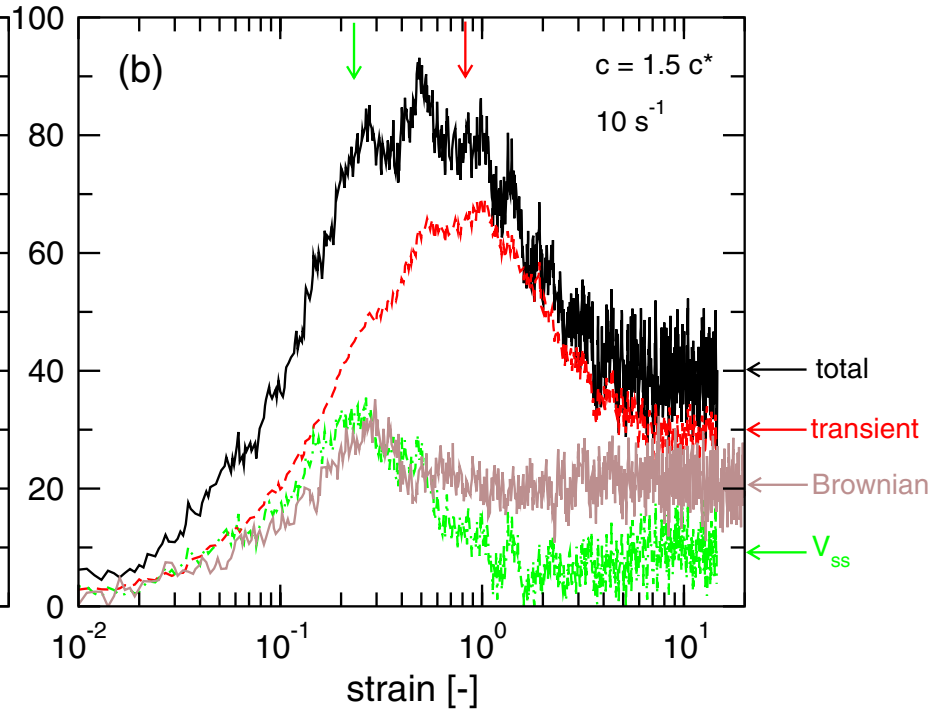

to conservative forces (green arrows at top scales) peaks sooner than that due to transient forces (red arrow). The (conservative) stress in a simulation without transient forces (solid brown line) shows a much weaker or no stress overshoot 
subjected to shear flow at a shear rate of $\dot{\gamma}=1 \mathrm{~s}^{-1}$ (a) or $\dot{\gamma}=10 \mathrm{~s}^{-1}$ (b). Note that the system is fluidised by the shear flow, effectively removing the non-ergodicity issues which apply to the (non-sheared) glassy state. The total stress (solid black line) may be partitioned into contributions arising from the conservative interactions $V_{s s}$ (dot-dashed green line) and those arising from transient interactions $\Phi_{t}$ (dashed red line). To avoid a possible misunderstanding, we do not refer to the contribution arising from the transient forces as a 'transient stress' because it does not decay to zero as the shear flow is maintained. This is caused by the fact that shear flow forces the entanglement numbers $n_{i j}$ to remain continually out of equilibrium.

At small strain $\gamma=\dot{\gamma} t$, both contributions to the stress increase almost linearly with $\gamma$. Then, both contributions display a stress overshoot before relaxing and settling to a steady-state value. An important difference is that the conservative contribution has its maximum at a strain of 0.2 to 0.3 , whereas the transient contribution has its maximum at a strain of order 1 . As a consequence, the maximum overshoot of the total shear stress occurs somewhere in between, although the noise in the data does not allow us to precisely pinpoint its location.

Figure 4 also shows the start-up shear stress measured in an equivalent Brownian simulation (solid brown line) in which the transient forces have been effectively disabled, thus leaving only the conservative forces. Clearly, the overshoot is absent for shear rate $1 \mathrm{~s}^{-1}$ and much weaker for $10 \mathrm{~s}^{-1}$. The peak for $10 \mathrm{~s}^{-1}$ appears at a strain of 0.3 , in agreement with the conservative part in the simulation with transient forces. Notice that, in both cases, for larger strain values, the conservative stress in the Brownian simulation is higher than the conservative stress in a simulation where transient forces are also present. This shows that the transient forces significantly change the path along which the system evolves under non-equilibrium conditions.

Figure 5 shows the start-up shear stress for all concentrations studied in this paper and shear rates ranging from $10^{-2}$ to $10^{2} \mathrm{~s}^{-1}$. A stress overshoot is observed in most cases. At the overlap concentration $c=c^{*}$, the maximum occurs at a strain of the order of 1 . For higher concentrations, the maximum occurs at lower strains, but its dependence on shear rate and concentration is complex. At fixed concentration, but with increasing shear rate, the location of the maximum moves to higher strain values, but only slightly. The concentration effect is much stronger. Although the noise in the data does not allow us to precisely pinpoint the locations of the maxima, Fig. 6 does suggest that, at fixed shear rate, the location of the maximum moves to lower strain values with increasing concentration. From a visual inspection of the curves, we estimate that probable ranges for the locations of the maxima are $\gamma_{\max } \in[0.8,2.0]$ for $c^{*}, \gamma_{\max } \in[0.6,1.1]$ for $1.2 c^{*}$ and $\gamma_{\max } \in[0.3,0.8]$ for $1.5 c^{*}$.

We are currently unable to perform simulations at higher densities (because of the increasingly large relaxation times involved), but experiments show that, for $c=2 c^{*}$, the maxima occur around a strain $\gamma_{\max }=$ 0.1 (Beris et al. 2008). This is in line with our observations. In this regard, it is important to note that the constitutive model for star polymer suspensions suggested by Beris et al. (2008) predicts locations of these maxima at strains that are one order of magnitude larger. This was attributed to the fact that the principal strain responsible for non-affine motion is located in the interfacial area between the particles, which amplifies
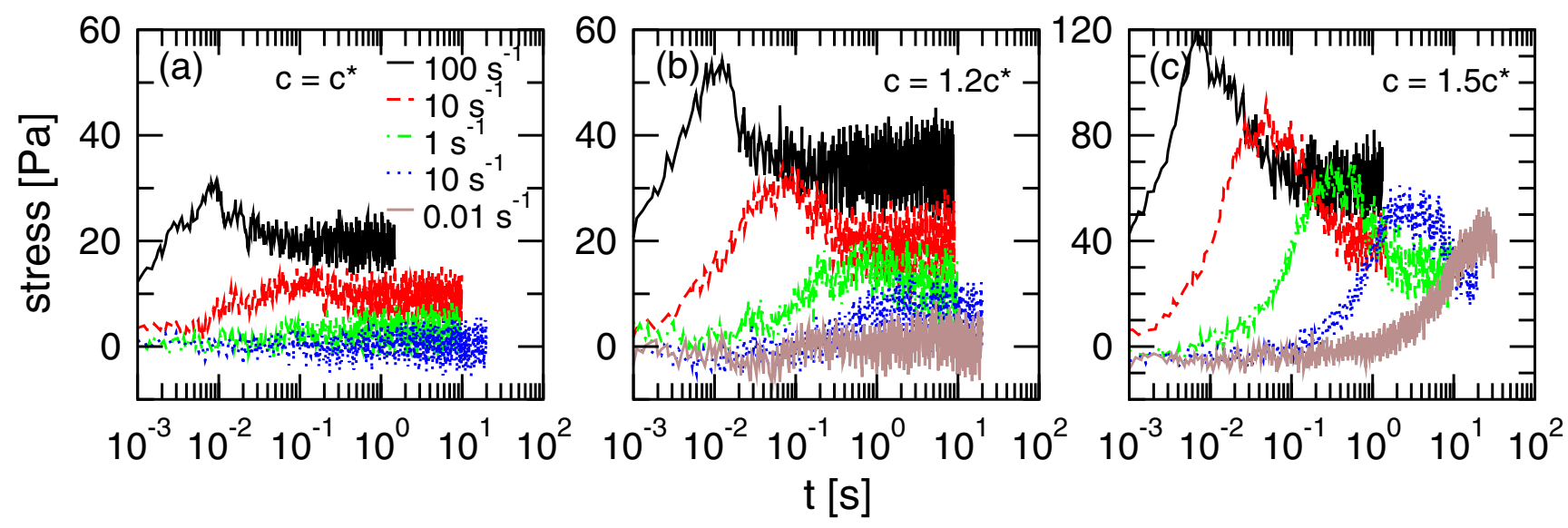

Fig. 5 Start-up shear stress for three different concentrations, $\mathbf{a} c=c^{*}, \mathbf{b} c=1.2 c^{*}$ and $\mathbf{c} c=1.5 c^{*}$, and different applied shear rates (see legend in a) 


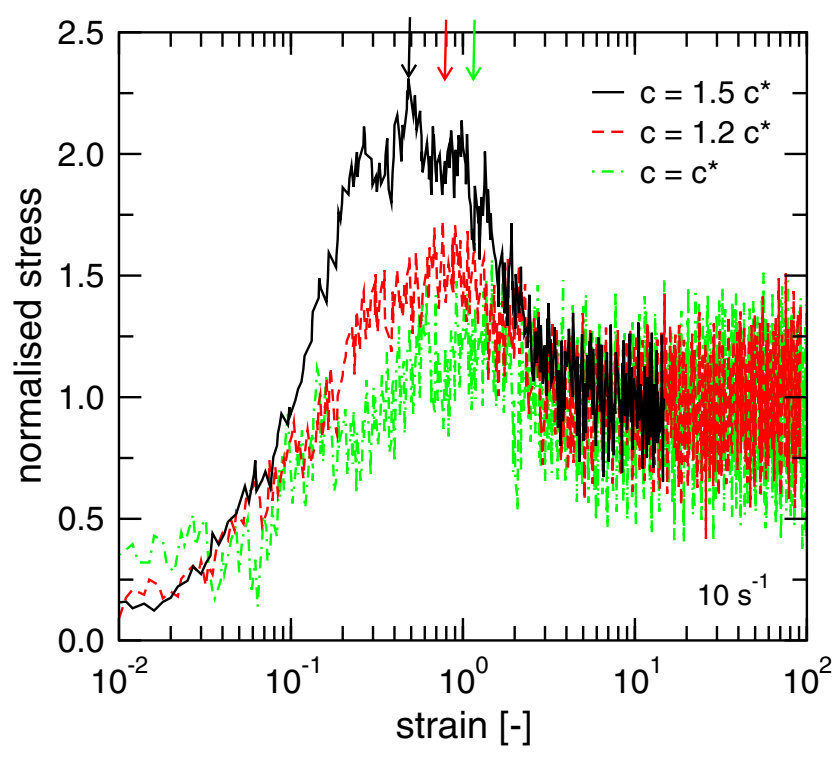

Fig. 6 Start-up shear stress normalised to its steady-state value for a shear rate of $\dot{\gamma}=10 \mathrm{~s}^{-1}$ and three different concentrations. Observe that the magnitude of the relative overshoot increases and that the strain where this maximum occurs (estimated strains are indicated by arrows at top scale) decreases with increasing concentration

the average strain present in the fluid. It is encouraging to see that the current simulation model yields qualitatively correct results without making such additional assumptions.
Interestingly, strain values $\gamma_{\max } \approx 0.1$ are also predicted for the positions of maximum stress overshoot in simulations of colloidal dispersions at the glass transition temperature, where the colloids interact only with relatively hard Yukawa potentials (Zausch et al. 2008) (and also in Lennard-Jones glasses (Varnik et al. 2004)). This suggests that, as the concentration of high-functionality star polymers is increased to inside the glass regime, the initial stress growth becomes increasingly dominated by the conservative interactions between the cores, rather than the entanglements between the arms.

Of course, the above does not mean that entanglements have no influence. Figure 4 shows that the stress contribution due to transient entanglement forces is dominant for strains larger than 0.2-0.3, with a maximum near strain 1 . This may be linked to the fact that the number of entanglements $n_{0}(r)$ changes only significantly over a distance of the order of one particle diameter $\sigma$, see Fig. 2, thus requiring a strain of order 1 for their influence to be fully felt.

\section{Cessation and step-down shear stress}

The relaxation of stress upon removal of the applied shear rate is shown against time in Fig. 7a for $c=$ $1.5 c^{*}$ and four different initially applied shear rates $\dot{\gamma}_{0}$.
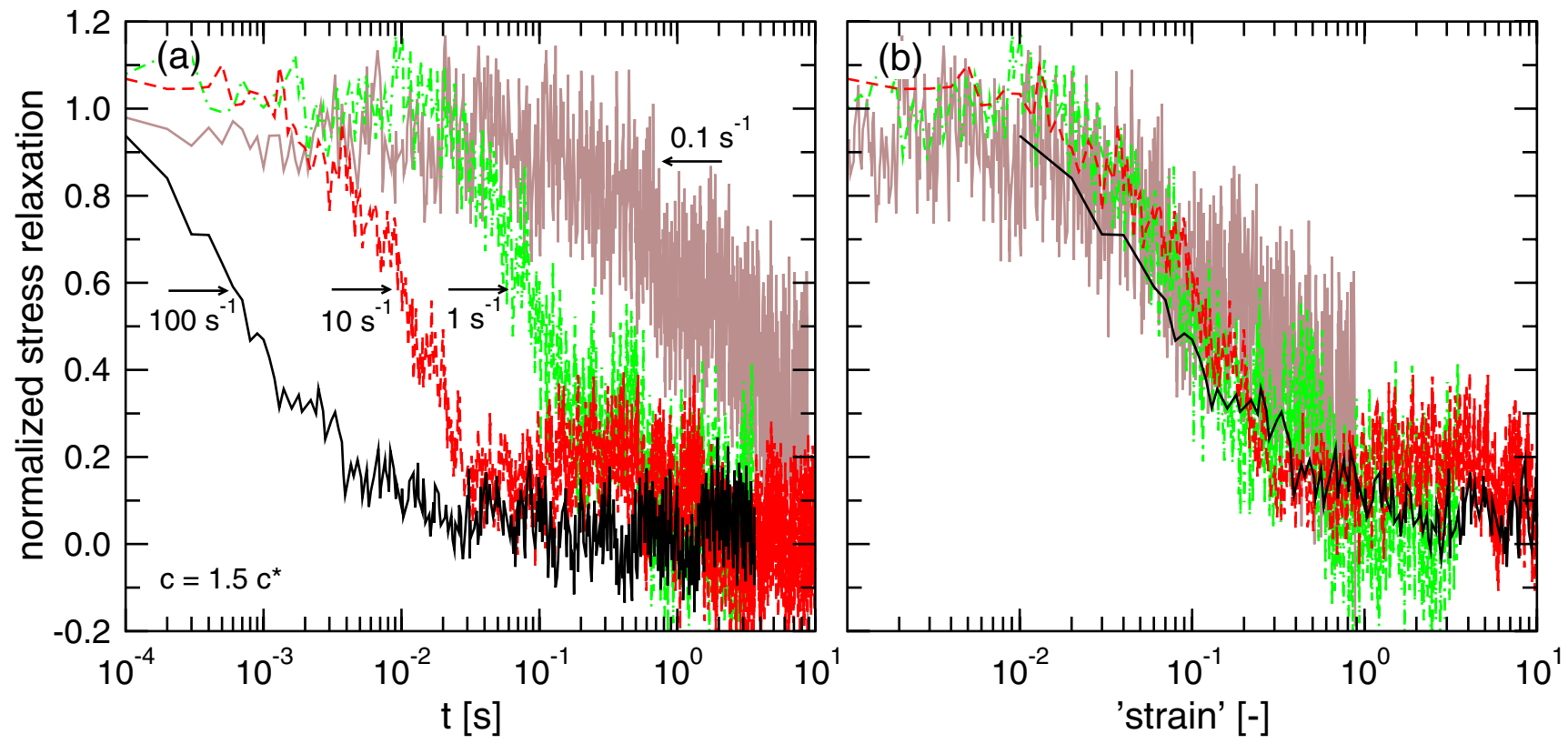

Fig. 7 Relaxation of shear stress (normalised to its previous steady-state value) upon removal of imposed shear rate at time $t=0$ for a star polymer suspension at a concentration $c=1.5 c^{*}$. The initially applied shear rate $\dot{\gamma}_{0}$ ranges from 0.1 to $100 \mathrm{~s}^{-1}$. The relaxation is shown against the time $t$ in a and against $\dot{\gamma}_{0} t$ in $\mathbf{b}$. The latter is the strain the system would have acquired if the shear would have continued with the initial shear rate 
Again, note that, for all these shear rates, the $1.5 c^{*}$ system is fluidised by the shear flow, overcoming the non-ergodicity issues which apply to the glassy state in equilibrium. For a fluid in the linear regime, the time dependence of the stress relaxation is given by the (linear) shear relaxation modulus $G(t)$. This means that the normalised stress relaxation curves for all previously applied shear rates would coincide. This is clearly not the case here (remember that the linear shear relaxation modulus for $1.5 c^{*}$ hardly decays over the entire time scale of the simulation). The relaxation is non-linear for all shear rates studied, and it is faster when the previously applied shear rate was higher. In the literature, this is attributed to the ageing of the system and its rejuvenation by shear flow (Beris et al. 2008; Bonn et al. 2004; McKenna et al. 2009; Moller et al. 2006; Rogers et al. 2008). Our observations are very similar to the experimental results shown in Figure 7 of reference Beris et al. (2008). In Fig. 7b we plot the relaxation against $\dot{\gamma}_{0} t$, which is the strain the system would have acquired if the shear would have continued with the initial shear rate $\dot{\gamma}_{0}$. In all cases the main relaxation takes place for 'strain' values between 0.01 and 1 . The data more or less collapse, but it must be noted that slower relaxation modes become visible for lower $\dot{\gamma}_{0}$. For low initial shear rates, the fluidised system has more time to return to the glassy state before all stress has relaxed.
Instead of completely stopping the applied shear rate, one may also step down the applied shear rate to a lower value at time. Figure $8 \mathrm{a}$ shows the shear stress at time $t$ after such a step-down to three new shear rates $\dot{\gamma}$, as well as the previous cessation $(\dot{\gamma}=0)$ experiment, for $c=1.5 c^{*}$ and an initial shear rate of $\dot{\gamma}_{0}=100 \mathrm{~s}^{-1}$. Initially, the stress decay is the same for all cases and follows the cessation experiment. In each particular case, the stress departs from this curve at a point where it has reached a value below the steadystate value belonging to the new shear rate. This causes an apparent minimum in the shear stress curve. Figure $8 \mathrm{~b}$ shows that the point of departure is after a strain of approximately 0.05 to 0.1 , where this strain value is based on the new shear rate. To our knowledge, this has not yet been tested experimentally.

\section{Steady-state shear stress (flow curves)}

When shear is applied for a sufficiently long time, a steady state is achieved. Figure 9 shows the steadystate shear stress as a function of shear rate (the socalled flow curve) for all concentrations studied in this work. For $c=c^{*}$ and $c=1.2 c^{*}$ and low shear rates, the shear stress is linearly dependent on the applied shear rate. The magnitude of this linear response is in agreement with the zero-shear viscosity obtained by
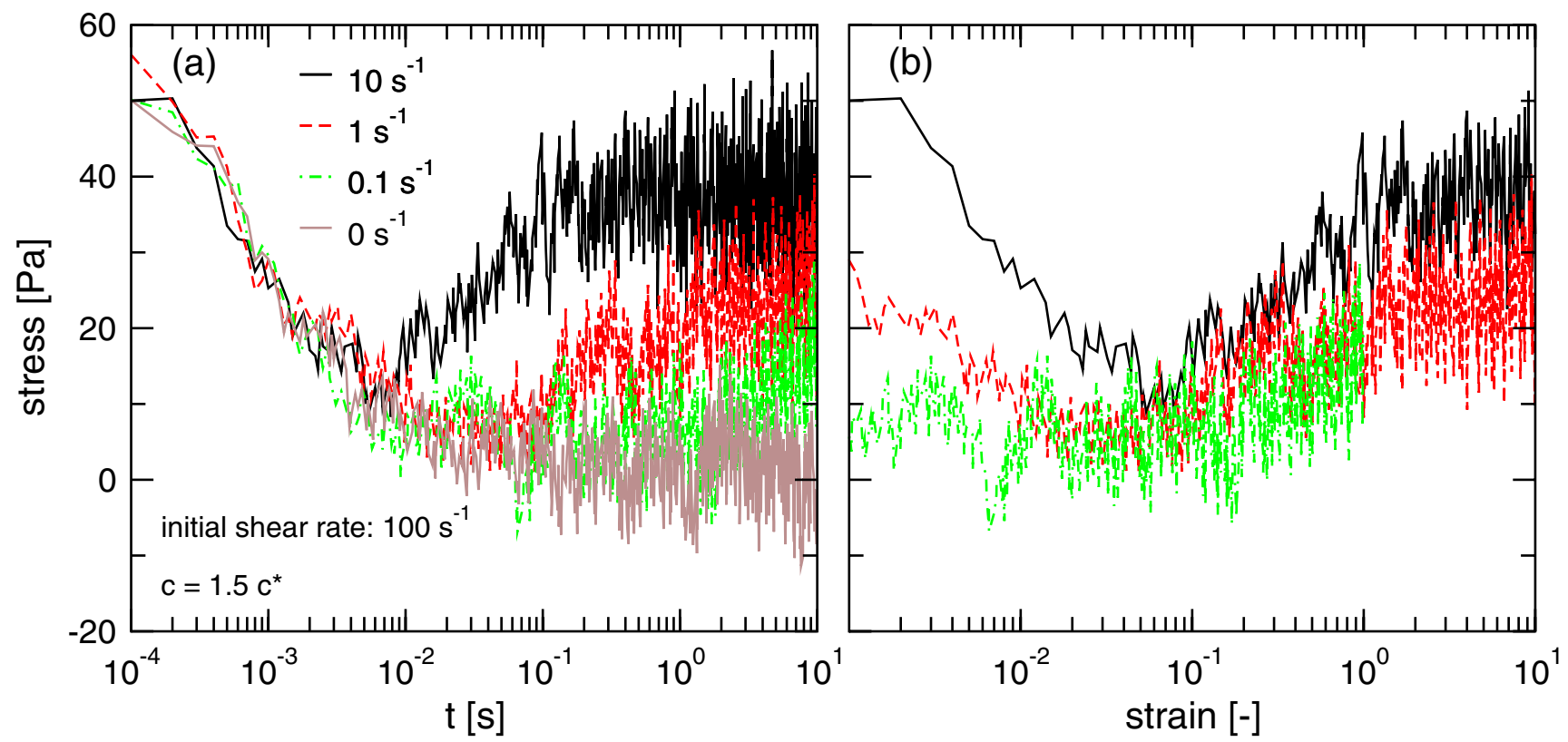

Fig. 8 Evolution of shear stress after stepping down, at $t=0$, the applied shear rate from an initial $\dot{\gamma}_{0}=100 \mathrm{~s}^{-1}$ to a new $\dot{\gamma}=10$, 1 or $0.1 \mathrm{~s}^{-1}$ for a star polymer suspension at a concentration $c=1.5 c^{*}$. The evolution is shown against time $t$ in $\mathbf{a}$ and against the strain $\dot{\gamma} t$ in $\mathbf{b}$. For comparison, the stress relaxation upon removal of the applied shear rate $(\dot{\gamma}=0)$ is also shown in a 


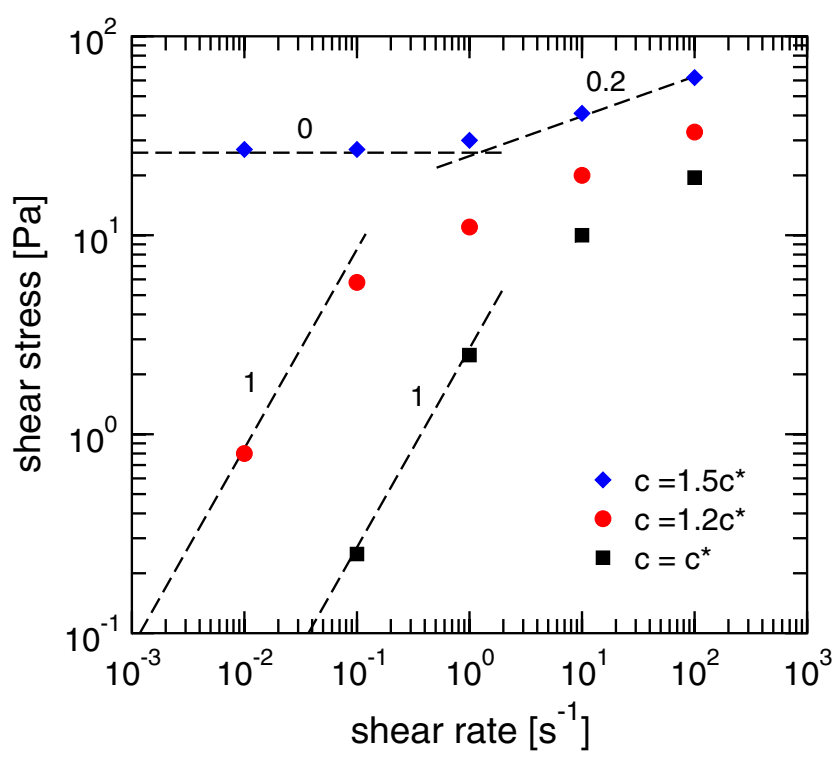

Fig. 9 Double-logarithmic plot of the steady-state shear stress of star polymer suspensions $(f=128)$ as a function of applied shear rate for all concentrations studied. Dashed lines indicated by their slope ' 1 ' are not fits, but show the linear prediction $\sigma_{x y}=\eta_{0} \dot{\gamma}$, where the zero-shear viscosity $\eta_{0}$ is obtained by integrating the shear relaxation modulus $G(t)$ (see Fig. 3). At $c=1.5 c^{*}$, no linear response can be found within the range of shear rates studied. For this high concentration, the shear stress is constant for shear rates lower than $1 \mathrm{~s}^{-1}$

integrating the shear relaxation modulus of Fig. 3. For $c=1.5 c^{*}$, no linear response can be found within the range of shear rates studied. At this high concentration, the shear stress shows a plateau for shear rates lower than $1 \mathrm{~s}^{-1}$, and increases slowly like $\sigma_{x y} \propto \dot{\gamma}^{n}$ with an exponent $n \approx 0.2$ for higher shear rates (note that the slow increase at higher shear rates is nearly identical for all three concentrations). In simulations without transient forces, the stress magnitudes are all lower than their counterparts from the simulations which do include transient forces (not shown). More importantly, no stress plateau is observed. Without transient forces, all stress values for $c=1.5 c^{*}$ can be fit to $\sigma_{x y} \propto \dot{\gamma}^{n}$ with $n \approx 0.32$, in the full range of shear rates studied.

A stress plateau may be associated with shear banding (non-linear shear velocity profiles) and with yield stress in the star polymer system (Fielding et al. 2009; Moller et al. 2006; Rogers et al. 2008). However, we do not observe the formation of shear bands in the flow profile, even though the simulated equations of motion do not bias towards a linear profile by construction (van den Noort and Briels 2008; Sprakel et al. 2009). Shear banding may be suppressed in our simulations because of the relatively small size of the simulation box, or it may occur only at times much longer than simulated here (Rogers et al. 2008).
A plateau and a slow increase in stress are qualitatively similar to experimental observations reported for $c=2 c^{*}$ in Figure 5 of Beris et al. (2008), although, experimentally, the exponent for the slow stress increase is $n \approx 0.3$ rather than $n \approx 0.2$ in the first two decades after the plateau region. Given the gross simplifications made in modelling the star polymer suspensions, we regard this as a very satisfying result. In fact, the predicted magnitude (at $c=1.5 c^{*}$ ) of approximately $30 \mathrm{~Pa}$ for the stress plateau (or yield stress) is very close to the experimental plateau value (at $c=2 c^{*}$ ) of approximately $8 \mathrm{~Pa}$ (Beris et al. 2008). It must be noted that, in these experiments, the plateau in the stress could only be observed if the shear was applied for a sufficiently long time. These experiments used a pre-shearing protocol, in which the system was brought into a fluidised state by large-amplitude oscillatory shear before commencing the constant shear rate experiment. As a consequence, the shear stress first attained a lower value and only after a long time, depending on the sample volume fraction and the experimental conditions (such as sample shear history), the stress jumped to the plateau value. Here we have not applied such a fluidising pre-shearing protocol, which explains why we are able to measure the plateau value after a relatively short time. Moreover, it is possible that the time needed for rearrangements in the system depends on the system size; our simulated system size is many orders of magnitude smaller than that of a sample in a rheometer. This is a topic of future research.

\section{Conclusion and outlook}

We have presented a novel particle-based simulation of the rheology of concentrated suspensions of star polymers with high functionality and long arms. This has been made possible by an extreme form of coarsegraining in which each star polymer is represented by a single particle. In order to faithfully reproduce dynamical properties with such a coarse model, it is very important to not only include time-averaged interactions (potentials of mean force), but to also account for transient interactions induced by entanglements between the arms of the star polymers.

The parameters of the potential of mean force, Eq. 2, are based on previous theoretical and simulation work, combined with knowledge of the number of arms and the experimentally determined hydrodynamic radius. In the absence of detailed experimental data, most of the other simulation parameters have been estimated in a rather crude way. The functional dependence of the number of entanglements on the separation distance 
is modelled by the overlap integral of two monomer density profiles. Finally, the parameters $\alpha, \tau$ and $\xi_{e}$ have been used as fitting parameters to match the experimentally determined zero-shear viscosity at overlap concentration $c^{*}$, an estimated self-diffusion coefficient at $c^{*}$ and the fact that overshoots in the start-up shear stress at $1.5 c^{*}$ are observed for shear rates $\geq 0.005 \mathrm{~s}^{-1}$. We emphasise that the output is not simply what we put in; the model predicts new features, many of which are in agreement with experimental observations on concentrated suspensions star polymers with high functionality and long arms:

- Without flow, the system gets kinetically trapped in a glassy state when the concentration is $1.5 c^{*}$ (or higher).

- For sufficiently high shear rates, the start-up shear stress displays an overshoot. With increasing concentration, the 'core' interactions increasingly dominate the initial stress response, leading to a maximum in the stress overshoot at a strain as small as $\approx 0.5$ for $c=1.5 c^{*}$ and 0.1 for $c=2 c^{*}$. The transient forces start to dominate the stress response after this initial stage.

- The relaxation of stress after cessation of shear flow is faster if the previously applied shear rate was higher. This is linked to the ageing of the system and its rejuvenation by shear flow.

- If the applied shear rate is suddenly stepped down to a lower but finite value, the shear stress initially follows the curve of the stopping experiment. After a strain of 0.05 to 0.1 (based on the new shear rate), the stress departs from this curve and tends to the new steady-state value, leaving an apparent undershoot.

- The steady-state shear stress shows a plateau for $c=1.5 c^{*}$ at sufficiently low shear rates. Without transient forces, this plateau is absent.

There is lots of room for improvement of the simulation method. Let us give an outlook on a few possible future developments. The approximation of a single relaxation time for the transient forces is probably too strict. One can imagine that entanglements between the arms of relatively distant star polymers relax faster than those of close star polymers, simply because effectively less chain length is available for (dis)entanglement. An alternative view is that the time for structural rearrangements in the star positions should increase as the stars are packed closer together. In both interpretations, the effect may be included by introducing a relaxation time $\tau\left(r_{i j}\right)$, which decreases with increasing pair-distance $r_{i j}$.
Furthermore, we have ignored the deformability of the stars under shear flow. Indeed, there is evidence that a single star will deform, albeit slightly, under strong shear (Ripoll et al. 2006). Our promising results will trigger further improvements in the future, including incorporation of deformability.

Another possible deficiency of the model is also related to deformability and the rather low polymer concentration, even when $c=2 c^{*}$. It may be easier for two high-functionality star polymers to indent and deform each other than to overlap and entangle their arms. On approaching each other, both stars will still feel a transient repulsion because the instantaneous distribution of monomeric mass in the particles will (temporarily) differ from the equilibrium one. However, on separation, it is unlikely that attractive forces will be operational. With this deficiency in the model, it is unlikely that full quantitative agreement can be achieved with the experimental high-functionality $(f=128)$ star polymer suspension rheology.

The indentation and deformation dominance will be less for highly entangled, but lower-functionality, star polymers. The model described in this paper may therefore be more accurate for star polymers with very long arms and $f$ in the range of 10 to 50 . Both simulations and experiments on such systems are planned for the near future.

Acknowledgements We thank Peter Kindt for useful discussions. The authors thank the NoE 'SoftComp' and NMP SMALL 'Nanodirect' for financial support. JTP thanks the Netherlands Organisation for Scientific Research (NWO) and EvR thanks FNRS - Communauté Française de Belgique for additional financial support.

Open Access This article is distributed under the terms of the Creative Commons Attribution Noncommercial License which permits any noncommercial use, distribution, and reproduction in any medium, provided the original author(s) and source are credited.

\section{References}

Allen MP, Tildesley DJ (1987) Computer simulation of liquids. Clarendon, Oxford

Beris AN, Stiakakis E, Vlassopoulos D (2008) A thermodynamically consistent model for the thixotropic behavior of concentrated star polymer suspensions. J Non-Newton Fluid Mech 152:76-85

Bonn D, Tanaka H, Coussot P, Meunier J (2004) Ageing, shear rejuvenation and avalanches in soft glassy materials. J Phys Condensed Matter 16:S4987-S4992

Briels WJ (2009) Transient forces in flowing soft matter. Soft Matter 5:4401-4411

Daoud M, Cotton JP (1982) Star shaped polymers-a model for the conformation and its concentration-dependence. J Physique 43:531-538 
Fielding SM, Cates ME, Sollich P (2009) Shear banding, aging and noise dynamics in soft glassy materials. Soft Matter $5: 2378-2382$

Furukawa T, Ishizu K, Yamane Y, Ando I (2005) Diffusional behavior of multi-arm star polymers by H-1 pulsed field gradient spin-echo NMR method. Polymer 46:1893-1898

Grest GS, Kremer K, Witten TA (1987) Structure of many-arm star polymers-a molecular-dynamics simulation. Macromolecules 20:1376-1383

Helgeson ME, Wagner NJ, Vlassopoulos D (2007) Viscoelasticity and shear melting of colloidal star polymer glasses. J Rheol 51:297-316

Huissmann S, Blaak R, Likos CN (2009) Star polymers in solvents of varying quality. Macromolecules 42:2806-2816

Jusufi A, Watzlawek M, Löwen H (1999) Effective interaction between star polymers. Macromolecules 32:4470-4473

Jusufi A, Dzubiella J, Likos CN, von Ferber C, Löwen H (2001) Effective interactions between star polymers and colloidal particles. J Phys, Condens Matter 13:6177-6194

Kindt P, Briels WJ (2007) A single particle model to simulate the dynamics of entangled polymer melts. J Chem Phys 127:134901

Laurati M, Stellbrink J, Lund R, Richter D, Zaccarelli E (2005) Starlike micelles with starlike interactions: a quantitative evaluation of structure factors and phase diagram. Phys Rev Lett 94:195504

Likos CN (2006) Soft matter with soft particles. Soft Matter 2:478-498

Likos CN, Löwen H, Watzlawek M, Abbas B, Jucknischke O, Allgaier J, Richter D (1998) Star polymers viewed as ultrasoft colloidal particles. Phys Rev Lett 80:4450-4453

Mayer C, Likos CN (2007) A coarse-grained description of starlinear polymer mixtures. Macromolecules 40:1196-1206

McKenna GB, Narita T, Lequeux F (2009) Soft colloidal matter: a phenomenological comparison of the aging and mechanical responses with those of molecular glasses. J Rheol 53: 489-516

Moller PCF, Mewis J, Bonn D (2006) Yield stress and thixotropy: on the difficulty of measuring yield stresses in practice. Soft Matter 2:274-283

Padding JT, Boek ES, Briels WJ (2008) Dynamics and rheology of wormlike micelles emerging from particulate computer simulations. J Chem Phys 129:074903

Pakula T (1998) Static and dynamic properties of computer simulated melts of multiarm polymer stars. Comput Theor Polymer Sci 8:21-30

Pakula T, Vlassopoulos D, Fytas G, Roovers J (1998) Structure and dynamics of melts of multiarm polymer stars. Macromolecules 31:8931-8940

Ripoll M, Winkler RG, Gompper G (2006) Star polymers in shear flow. Phys Rev Lett 96:188302
Rissanou AN, Yiannourakou M, Economou IG, Bitsanis IA (2006) Temperature-induced crystallization in concentrated suspensions of multiarm star polymers: a molecular dynamics study. J Chem Phys 124:044905

Rogers SA, Vlassopoulos D, Callaghan PT (2008) Aging, yielding, and shear banding in soft colloidal glasses. Phys Rev Lett 100:128304

Roovers J (1994) Concentration-dependence of the relative viscosity of star polymers. Macromolecules 27:53595364

Roovers J, Zhou L-L, Toporowski TM, van der Zwan M, Iatrou H, Hadjichristidis N (1993) Regular star polymers with 64 and 128 arms - models for polymeric micelles. Macromolecules 26:4324-4331

Sprakel J, Spruijt E, van der Gucht J, Padding JT, Briels WJ (2009) Failure-mode transition in transient polymer networks with particle-based simulations. Soft Matter 5: 4748-4756. doi:10.1039/b910425a

Stiakakis E, Vlassopoulos D, Loppinet B, Roovers J, Meier G (2002a) Kinetic arrest of crowded soft spheres in solvents of varying quality. Phys Rev E 66:051804

Stiakakis E, Vlassopoulos D, Likos CN, Roovers J, Meier G (2002b) Polymer-mediated melting in ultrasoft colloidal gels. Phys Rev Lett 89:208302

van den Noort A, Briels WJ (2007) Coarse-grained simulations of elongational viscosities, superposition rheology and shear banding non model core-shell systems. Macromol Theory Simul 16:742-754

van den Noort A, Briels WJ (2008) Brownian dynamics simulations of concentration coupled shear banding. J Non-Newton Fluid Mech 152:148-155

van den Noort A, den Otter WK, Briels WJ (2007) Coarse graining of slow variables in dynamic simulations of soft matter. Euro Phys Lett 80:28003

Varnik F, Bocquet L, Barrat J-L (2004) A study of the static yield stress in a binary Lennard-Jones glass. J Chem Phys 120:2788-2801

Vlassopoulos D (2004) Colloidal star polymers: models for studying dynamically arrested states in soft matter. J Polym Sci, B, Polym Phys 42:2931-2941

von Ferber C, Jusufi A, Likos CN, Löwen H, Watzlawek M (2000) Triplet interactions in star polymer solutions. Eur Phys J E 2:311-318

Watzlawek M, Likos CN, Löwen H (1999) Phase diagram of star polymer solutions. Phys Rev Lett 82:5289-5292

Witten TA, Pincus PA (1986) Colloid stabilization by long grafter polymers. Macromolecules 19:2509-2513

Zausch J, Horbach J, Laurati M, Egelhaaf SU, Brader JM, Voigtmann Th, Fuchs M (2008) From equilibrium to steady state: the transient dynamics of colloidal liquids under shear. J Phys, Condens Matter 20:404210 